\title{
Multiview and multifeature spectral clustering using common eigenvectors
}

\section{Samir Kanaan-Izquierdo*, ac}

samir.kanaan@upc.edu

Andrey Ziyatdinov

Alexandre Perera-Lluna $a^{a, c}$

${ }^{\mathrm{a} C e n t r e ~ d e ~ R e c e r c a ~ e n ~ E n g i n y e r i a ~ B i o m e ̀ d i c a, ~ U n i v e r s i t a t ~ P o l i t e ̀ c n i c a ~ d e ~ C a t a l u n y a, ~ P a u ~ G a r g a l l o ~ 5, ~ B a r c e l o n a, ~ 08028, ~ S p a i n ~}$

bDepartment of Epidemiology, Harvard T.H. Chan School of Public Health, Boston, Massachusetts, United States

${ }^{\circ}$ CIBER of Bioengineering, Biomaterials and Nanomedicine (CIBER-BBN), Barcelona, Catalonia, Spain

*Corresponding author at: Centre de Recerca en Enginyeria Biomèdica, Universitat Politècnica de Catalunya. Pau Gargallo 5. Barcelona, 08028, Spain. Fet.: +34 934137389;fax: +34-93 41374 00

\section{Abstract}

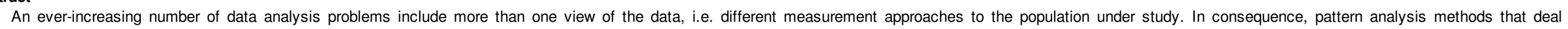

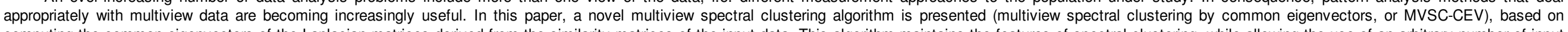

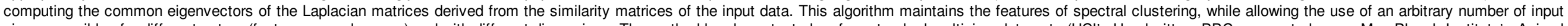

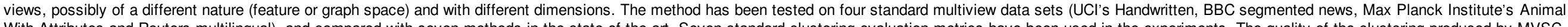

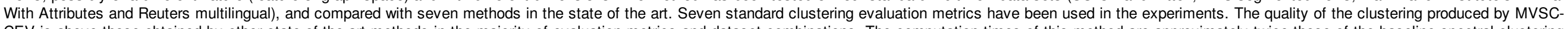

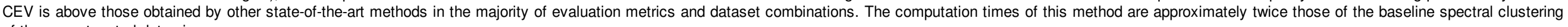
of the concatenated data views.

Keywords: Multiview data; Spectral clustering; Common eigenvectors

MSC: 41A05; 41A10; 65D05; 65D17

\section{Introduction}

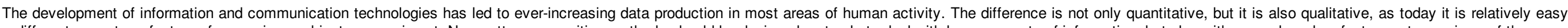

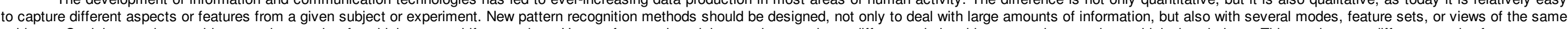

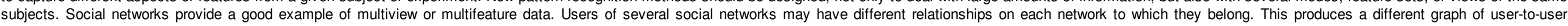

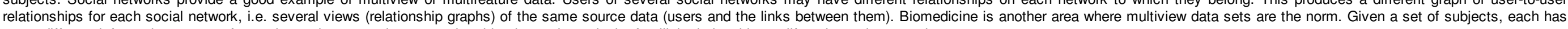
many different information aspects for study, such as genetic sequencing, blood sample analysis, familial relationships to lifestyle, and many others.

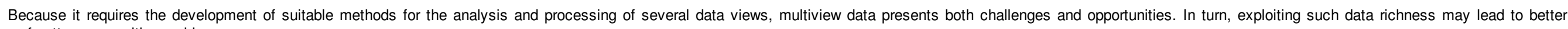
resolution of pattern recognition problems.

\subsection{Multiview clustering interpretation}

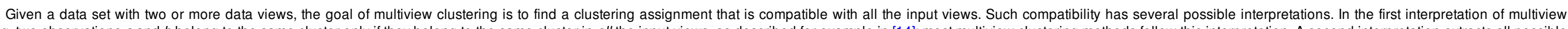

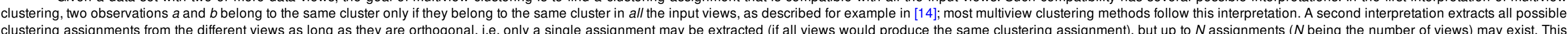
approach is described in [6]. Yet another possible interpretation assigns observations $a$ and $b$ to the same cluster if they belong to the same cluster in some of the input views; the method presented in this paper follows this interpretation.

\subsection{Multiview clustering methods}

In recent years, several multiview clustering approaches have been developed. In this section, we briefly describe them in order to provide a context for the algorithm presented in this paper.

In general terms, clustering multiple data views $V_{1}, V_{2}, \ldots V_{c}$ involves the following steps:

1. Obtain a similarity matrix $S_{i}$ for each view $V_{i}$

2. Compute a projection $P_{i}$ of each $S_{i}$ into a space suitable for clustering.

3. Produce a clustering assignment.

The main structural difference between the multiview clustering methods proposed in the literature lies in the step where the information from the multiple views is collapsed into a single view to produce the final clustering assignment.

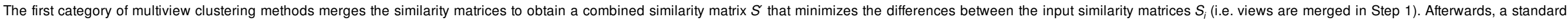

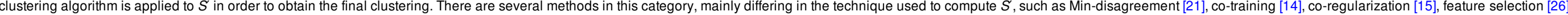


or graph fusion [17].

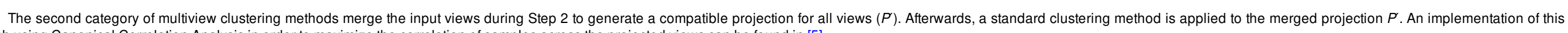
approach using Canonical Correlation Analysis in order to maximize the correlation of samples across the projected views can be found in [5]

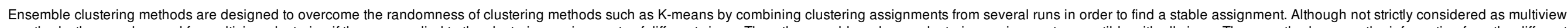

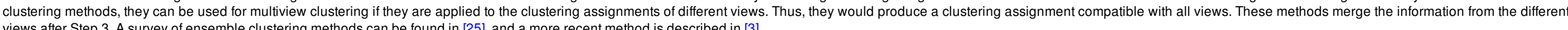
views after Step 3. A survey of ensemble clustering methods can be found in [25], and a more recent method is described in [3].

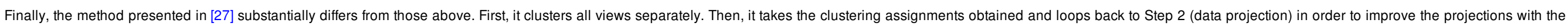
clustering information previously obtained. Therefore it is a co-training approach, since it uses the results of one iteration to further improve the results of the final clustering.

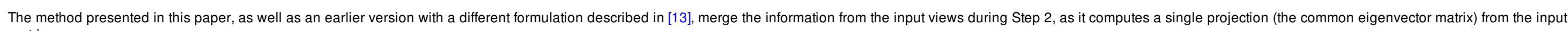
similarity matrices.

\subsection{Goal of the present work}

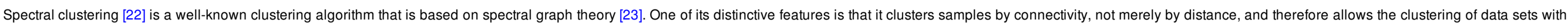
concave or nested clusters.

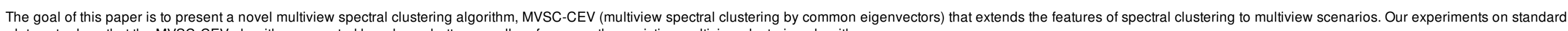
multiview data sets show that the MVSC-CEV algorithm presented here has a better overall performance than existing multiview clustering algorithms.

\subsection{Structure of this paper}

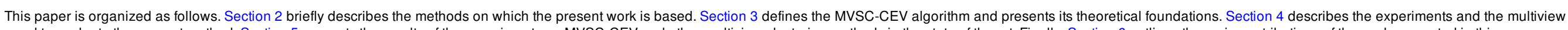

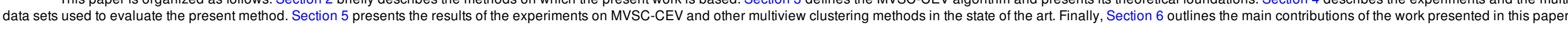

\section{Related work}

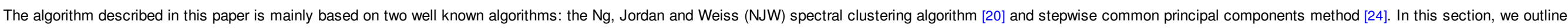
both algorithms in order to provide a theoretical background for the MVSC-CEV algorithm described in Section 3.

\subsection{Spectral clustering}

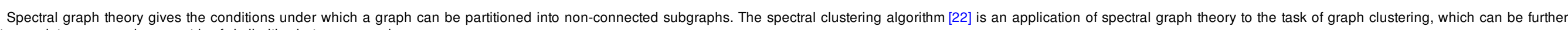
applied to any data expressed as a matrix of similarities between samples.

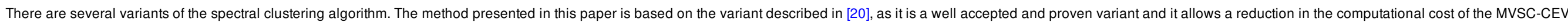
algorithm, as will be explained in Section 3. As a reference for the definition of MSCV-CEV on Section 3, the NJW spectral clustering algorithm is shown in Algorithm 1.

\section{Input: a set of data samples $P=\left\{p_{1}, \ldots, p_{n}\right\}, \sigma$ for the Gaussian similarity function, and the number $k$ of desired clusters.}

1. Build a similarity matrix $S \in \mathbb{R}^{n \times n}$ from $P$ using the Gaussian similarity function $G(x, y)=\exp \left(-\|x-y\|^{2} / 2 \sigma^{2}\right)$, so that $S_{i j}=G\left(p_{i}, p_{j}\right)$ if $i \neq j$, else $S_{i j}=0$.

2. Construct the normalized symmetrical Laplacian matrix $L=D^{-1 / 2} S D^{-1 / 2}$, where $D$ is the diagonal matrix whose $(i, i)$ element is the sum of the $i$ th row of $S$

3. Create a matrix $X \in \mathbb{R}^{n x k}$ with the $k$ largest eigenvectors of $L$ disposed in columns.

4. Normalize $X$ so that each row has unit length, obtaining $Y \in \mathbb{R}^{n \times d}$.

5. Apply K-means or another clustering algorithm to $Y$.

Output: a clustering assignment in $k$ clusters of the $n$ samples in $P$.

Alanrithm 1 Snectral Eclustering - NJW variant

alt-text: Algorithm 1

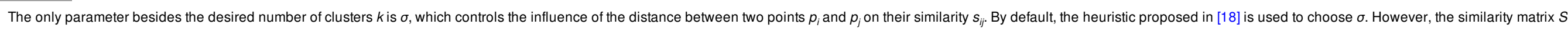
built in Step 1 can be obtained using a different similarity function.

Note that the algorithm is formulated to receive data in feature space (observations/features matrix) as input. However, it can also be applied to data in graph space (adjacency or similarity matrix).

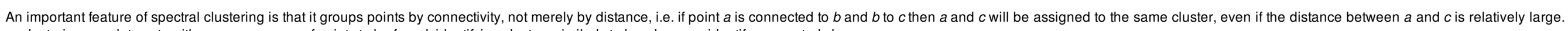
This allows clusterings on data sets with concave groups of points to be found, identifying clusters similarly to how humans identify connected shapes.

\subsection{Stepwise common principal components}

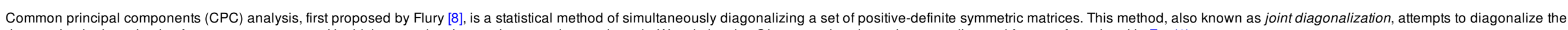
input matrices under the hypothesis of common components $H$, which states that there exists an orthogonal matrix Wsuch that the $C$ input matrices have the same diagonal form, as formulated in Eq. (1). 


\section{elsevier_PATREC_7027}

$H: L_{c}^{\prime}=W^{T} L_{c} W, c=1,2, \ldots, C$

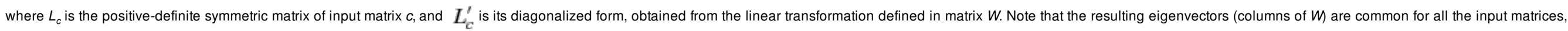

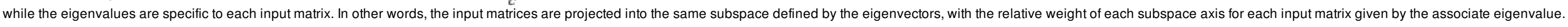

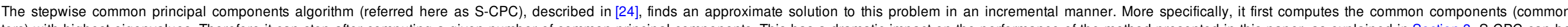

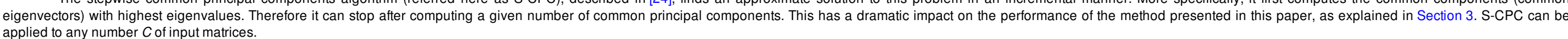

\section{Method: the MVSC-CEV algorithm}

\subsection{Description of the algorithm}

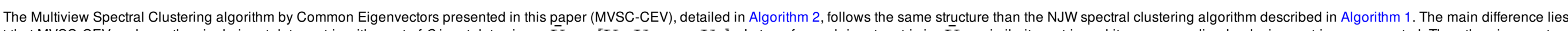

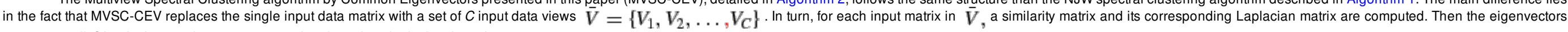
common to all $C$ Laplacian matrices are computed and used to obtain the clustering.

Input: $C$ view matrices $\bar{V}=\left\{V_{1}, V_{2}, \ldots, V_{C}\right\}$ of the data (with $n$ samples each), a vector $\left\{\sigma_{1}, \sigma_{2}, \ldots, \sigma_{C}\right\}$, and the number $k$ of

desired clusters.

1. For each data view $V_{c} \in \bar{V}$, compute a similarity matrix $S_{c} \in \mathbb{R}^{n \times n}$ using the Gaussian similarity function $G(x, y)=\exp (-\| x-$ $\left.y \|^{2} / 2 \sigma^{2}\right)$, so that $S_{c_{i j}}=G\left(v_{c_{i}}, v_{c_{j}}\right)$ if $i \neq j$, else $S_{c_{i j}}=0$, where $v_{c_{i}}$ is the $i$ th row of matrix $V_{c}$. The final result is a set of similarity matrices $\bar{S}=\left\{S_{1}, S_{2}, \ldots, S_{C}\right\}$

2. For each similarity matrix $S_{c} \in \bar{S}$ construct the normalized symmetrical Laplacian matrix $L_{c}=D^{-1 / 2} S D^{-1 / 2}$, where $D$ is the

diagonal matrix whose $(i, i)$ element is the sum of $S_{c}$ 's $i$ th row. The result is a set of Laplacian matrices $\bar{L}=\left\{L_{1}, L_{2}, \ldots, L_{C}\right\}$

3. Create a matrix $X \in \mathbb{R}^{n \times k}$ with the $k$ largest common eigenvectors of the matrices in $\bar{L}$, computed using the S-CPC algorithm

(Section 2.2).

4. Normalize $X$ so that each row has unit length, obtaining $Y \in \mathbb{R}^{n \times k}$.

5. Apply K-means or another clustering algorithm to $Y$.

Output: a single clustering assignment of the $n$ input data samples in $k$ clusters.

Alanrithm 2 Multiview spectral clustering by common eigenvectors (MVSC-CEV).

alt-text: Algorithm 2

MVSC-CEV can operate on both feature space and graph space input views, and on any combination of both. Input matrices in feature space can have any dimension, possibly varying across the different matrices.

Computational complexity The computational complexity of each step of Algorithm 2 is the following:

1. The cost of the Gaussian similarity function on input view $v_{c}$ is $O\left(n^{2} m_{c}\right)$, where $m_{c}$ is the number of columns of input view $v_{c}$. Thus the cost of computing the Gaussian similarity matrices on all the input views is $O\left(n^{2} M\right.$, where $M=\sum_{c=1}^{C} m_{c}$.

2. The total cost of computing the $C$ Laplacian matrices is $O\left(C \mathrm{C}^{2}\right)$.

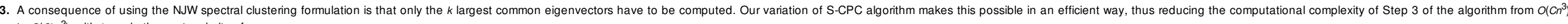
to $O\left(C k n^{2}\right)$, with $k \ll n$ in the vast majority of cases.

4. The cost of the normalization step is $O(k n)$.

5. Finally, the cost of K-means is $O\left(k n^{2}+t n^{2}\right)=O\left((k+t) n^{2}\right.$, where $t$ is the number of iterations $\mathrm{K}$-means is programmed to execute.

Adding the previous terms together gives the overall computational cost, $O\left((M+C+C k+k+t) n^{2}\right)$.

\subsection{Ideal clustering case}

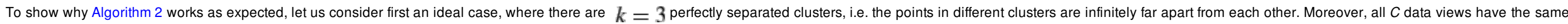

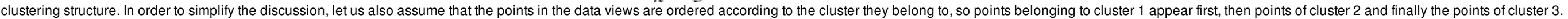

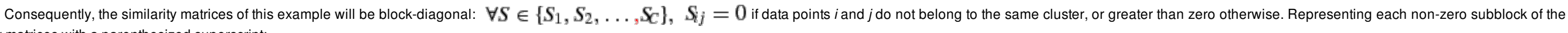
similarity matrices with a parenthesized superscript:

$S_{c}=\left[\begin{array}{ccc}S^{(1)} & 0 & 0 \\ 0 & S^{(2)} & 0 \\ 0 & 0 & S^{(3)}\end{array}\right] \forall S_{c} \in \bar{S}$

On the next step of the algorithm, for each similarity matrix $S_{c}$ a Laplacian matrix $L_{c}$ is computed, whose block-diagonal structure will be the same: 


\section{elsevier_PATREC_7027}

$L_{c}=\left[\begin{array}{ccc}L^{(1)} & 0 & 0 \\ 0 & L^{(2)} & 0 \\ 0 & 0 & L^{(3)}\end{array}\right] \forall L_{c} \in \bar{L}$

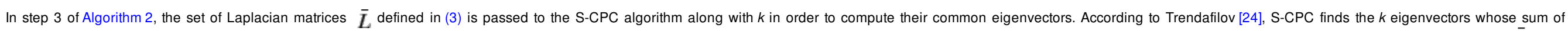

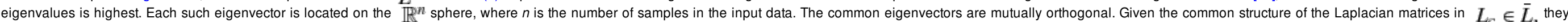

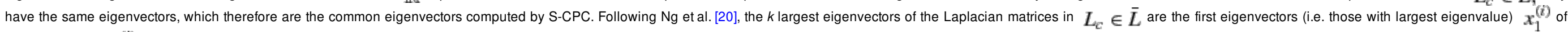
each submatrix $L_{c}^{(i)}$, properly padded with zeros to complete the missing elements:

$X=\left[\begin{array}{ccc}x_{1}^{(1)} & \overrightarrow{0} & \overrightarrow{0} \\ \overrightarrow{0} & x_{1}^{(2)} & \overrightarrow{0} \\ \overrightarrow{0} & \overrightarrow{0} & x_{1}^{(3)}\end{array}\right] \in \mathbb{R}^{n \times k}$

Finally, the normalization of the rows of $X$ to make them of unit length results in a matrix $Y$, of the form:

$Y=\left[\begin{array}{ccc}y^{(1)} & \overrightarrow{0} & \overrightarrow{0} \\ \overrightarrow{0} & y^{(2)} & \overrightarrow{0} \\ \overrightarrow{0} & \overrightarrow{0} & y^{(3)}\end{array}\right] \in \mathbb{R}^{n \times k}$

where $y^{(i)}$ is a vector of ones with as many values as the number of elements in cluster $i$. Applying K-Means to $Y$ produces the clustering assignment of the input data samples common to the $C$ input views.

\subsection{Deviations from the ideal case}

On multiview clustering problems there are two possible sources of deviation from the ideal case discussed in Section 3.2.

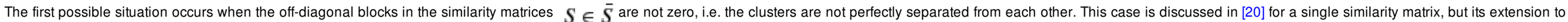
several similarity matrices with the same structure is straightforward.

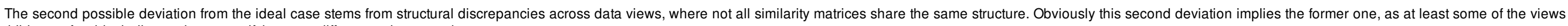
cannot exhibit a perfect block-diagonal structure if there are differences between them.

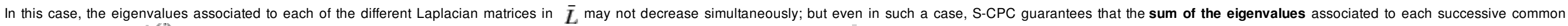
eigenvector is decreasing. Let $\lambda_{c}^{(i)}$ be the eigenvalue associated to Laplacian matrix $L_{c}$ obtained on iteration $i$ of S-CPC, i.e. associated with the $i$ th eigenvector. Therefore, the following relation holds:

$\sum_{c=1}^{C} \lambda_{c}^{(i)} \geq \sum_{c=1}^{C} \lambda_{c}^{(i+1)} \quad \forall i=1,2, \ldots, k$

in other words, the eigengaps (difference between consecutive eigenvalues) are conserved:

$\delta^{(i)}=\sum_{c=1}^{C} \lambda_{c}^{(i)}-\sum_{c=1}^{C} \lambda_{c}^{(i+1)} \geq 0 \quad \forall i=1,2, \ldots, k$

This satisfies the matrix perturbation theory condition [23], that guarantees the stability of the subspace defined by the first $k$ eigenvectors a matrix as long as the eigengaps are conserved.

\section{Experimental setup}

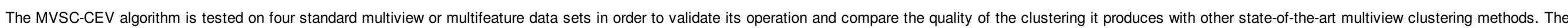
details of each data set, including the number and size of their data views, as well as the total number of classes and samples, are given in Table 1.

Table 1 Summarv of the data sets used in the experiments.

\begin{tabular}{|c|c|c|c|c|c|c|c|c|c|c|c|c|}
\hline \multirow[b]{2}{*}{ View \#. } & \multicolumn{3}{|c|}{ Digits } & \multicolumn{3}{|c|}{$\mathrm{BBC}$} & \multicolumn{3}{|c|}{ Reuters } & \multicolumn{3}{|c|}{ AWA } \\
\hline & View name & \# feat. & $\sigma$ & View name & \# feat. & $\sigma$ & View name & \# feat. & $\sigma$ & View name & \# feat. & $\sigma$ \\
\hline 1 & Pixels & 240 & 0.68476 & Segm. A & $500 / 6,838$ & 1.20467 & English & $500 / 21,531$ & 0.04631 & $C Q$ & 2,6882688 & 1.470858 \\
\hline
\end{tabular}




\section{elsevier_PATREC_7027}

\begin{tabular}{|c|c|c|c|c|c|c|c|c|c|c|c|c|}
\hline 2 & Fourier coeffs. & 76 & 0.61889 & Segm. B & $500 / 6,790$ & 1.20609 & French & $500 / 24,892$ & 0.05227 & LSS & $2,000 \underline{2000}$ & 0.075466 \\
\hline 3 & Profile correl. & 216 & 4.19278 & - & & & German & $500 / 34,251$ & 0.05443 & PHOG & 252 & 0.929560 \\
\hline 4 & Zernike coeffs. & 47 & 0.60180 & - & & & Italian & $500 / 15,506$ & 0.05506 & SIFT & $z, 000 \underline{2000}$ & 0.053216 \\
\hline 5 & Karhunen moments & 64 & 0.26484 & - & & & Spanish & $500 / 11,547$ & 0.05676 & RGSIFT & $2,000 \underline{2000}$ & 0.025925 \\
\hline 6 & Morph. feats. & 6 & 0.08377 & - & & & - & & & SURF & $2,000 \underline{2000}$ & 0.091183 \\
\hline \# samples & $2,000 \underline{2000}$ & & & $z, 1122112$ & & & $78758 \underline{18,758}$ & & & $30457 \underline{30,457}$ & & \\
\hline
\end{tabular}

If the number of features column has two numbers, the first is the number of features used and the second the total number of features in the dataset.

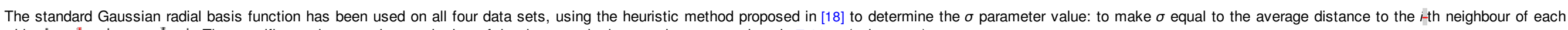
sample, with $i=\log \mid$ samples $\mid$. The specific $\sigma$ values used on each view of the data sets in the experiments are given in Table 1 (columns $\sigma$ ).

\subsection{Multiview datasets}

\subsubsection{Multiple features data set (Digits)}

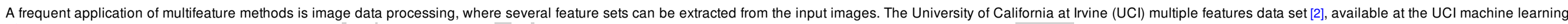

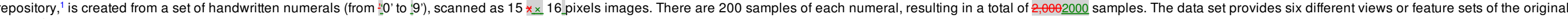
image data.

The input views do not require special preprocessing, and the similarity matrices are computed from the Euclidean distance matrix of each input view.

\subsubsection{BBC news (BBC)}

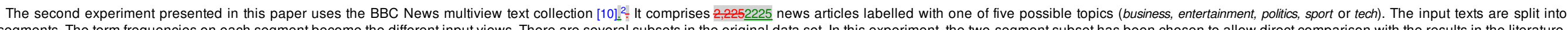

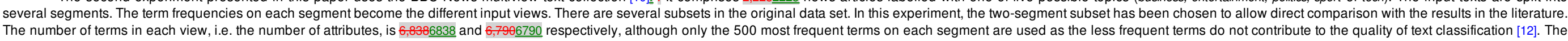
tf.idf (term frequency / inverse document frequency) [19] is computed on each of the input segments, and the cosine similarity is used instead of the euclidean distance because of the high sparsity of the feature matrices.

\subsubsection{Reuters multilingual corpus (Reuters)}

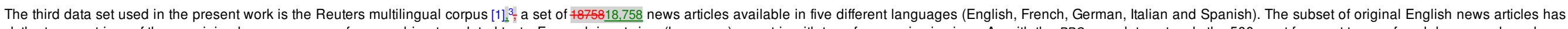

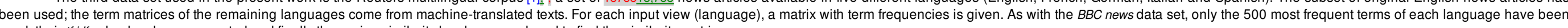
used, their tf.idf value has been computed and finally the cosine similarity has been employed to find the similarity matrices.

\subsubsection{Animal with attributes (AWA)}

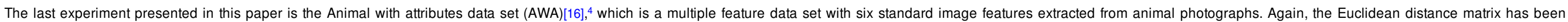
computed on the feature matrices and then passed to the Gaussian radial basis function.

\subsection{Evaluation metrics}

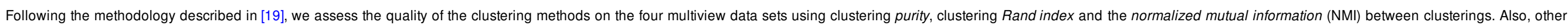
supervised clustering quality metrics used are clustering precision, recall and their $F$-score, according to the methodology followed in $[4,14,27]$ among others.

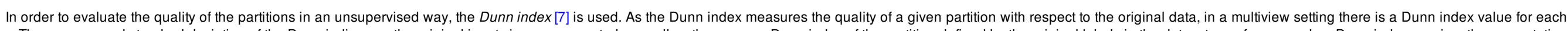

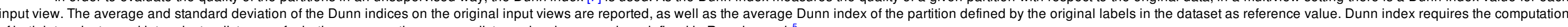
of both intra-cluster and inter-cluster distances; for both measures, the average distance has been used, as defined in $\mathrm{R}$ package $c / v^{5}$.

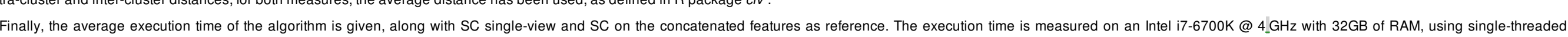

\section{Evaluation and results}

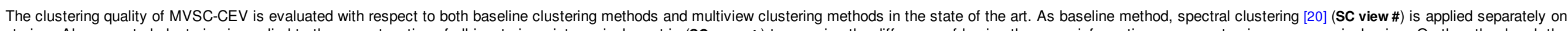

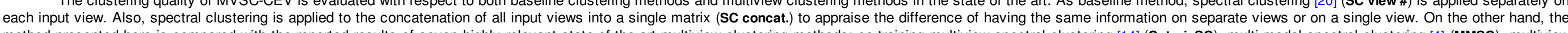

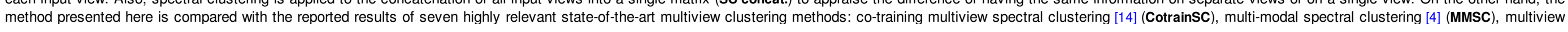




\section{elsevier_PATREC_7027}

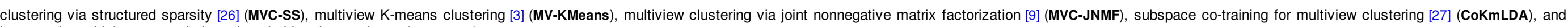
large-scale multiview spectral clustering via bipartite graph [17] (MVSC-BG).

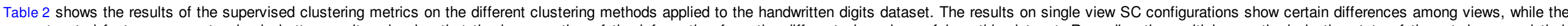

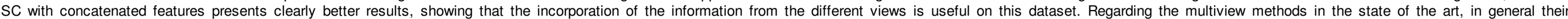
performance is below SC on concatenated features. However, MVSC-CEV produces the highest results in all six metrics, showing a better exploitation of the multiview data than the other methods.

Table 2 Clustering performance on the handwritten digits dataset.

\section{alt-text: Table 2}

\begin{tabular}{|c|c|c|c|c|c|c|}
\hline Method & F-score & Precision & Recall & NMI & Rand tindex & Purity \\
\hline SC view 1 & 0.615 & 0.611 & 0.620 & 0.671 & 0.568 & 0.721 \\
\hline SC view 2 & 0.532 & 0.466 & 0.619 & 0.632 & 0.467 & 0.651 \\
\hline SC view 3 & 0.587 & 0.573 & 0.602 & 0.660 & 0.536 & 0.697 \\
\hline SC view 4 & 0.644 & 0.621 & 0.669 & 0.709 & 0.599 & 0.750 \\
\hline SC view 5 & 0.495 & 0.462 & 0.533 & 0.562 & 0.430 & 0.610 \\
\hline SC view 6 & 0.634 & 0.597 & 0.677 & 0.685 & 0.587 & 0.744 \\
\hline SC concat. & 0.837 & 0.833 & 0.841 & 0.851 & 0.817 & 0.910 \\
\hline CotrainSC & 0.726 & 0.709 & 0.745 & 0.765 & 0.695 & $\mathrm{n} / \mathrm{a}$ \\
\hline MMSC & $\mathrm{n} / \mathrm{a}$ & $\mathrm{n} / \mathrm{a}$ & $\mathrm{n} / \mathrm{a}$ & 0.792 & $\mathrm{n} / \mathrm{a}$ & 0.758 \\
\hline MV-KMeans & $\mathrm{n} / \mathrm{a}$ & $n / a$ & $n / a$ & 0.807 & 0.789 & 0.825 \\
\hline MVC-JNMF & $\mathrm{n} / \mathrm{a}$ & $n / a$ & $n / a$ & 0.804 & 0.881 & $n / a$ \\
\hline CoKmLDA & $n / a$ & $n / a$ & $n / a$ & 0.818 & 0.819 & $n / a$ \\
\hline MVSC-BG & $\mathrm{n} / \mathrm{a}$ & $\mathrm{n} / \mathrm{a}$ & $\mathrm{n} / \mathrm{a}$ & 0.832 & $\mathrm{n} / \mathrm{a}$ & 0.844 \\
\hline MVSC-CEV & 0.899 & 0.897 & 0.900 & 0.892 & 0.886 & 0.946 \\
\hline
\end{tabular}

$n / a$ means result not available.

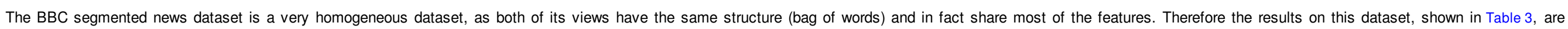

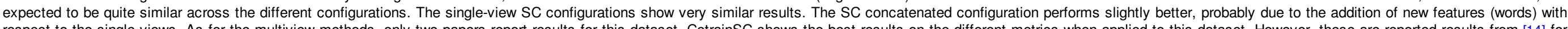

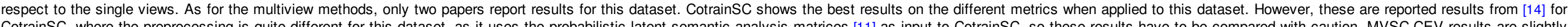

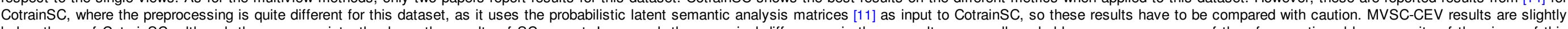

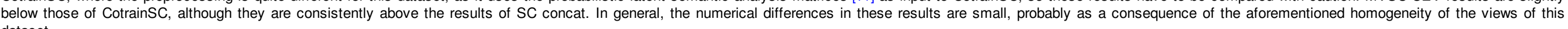
dataset.

Table 3 Clustering performance on the BBC segmented news dataset.

alt-text: Table 3

\begin{tabular}{|c|c|c|c|c|c|c|}
\hline Method & F-score & Precision & Recall & NMI & Rand tindex & Purity \\
\hline SC view 1 & 0.852 & 0.850 & 0.854 & 0.775 & 0.813 & 0.921 \\
\hline SC view 2 & 0.847 & 0.845 & 0.848 & 0.771 & 0.807 & 0.917 \\
\hline SC concat. & 0.871 & 0.867 & 0.875 & 0.810 & 0.837 & 0.932 \\
\hline CotrainSC & 0.898 & 0.894 & 0.902 & 0.841 & 0.873 & $\mathrm{n} / \mathrm{a}$ \\
\hline CoKmLDA & $\mathrm{n} / \mathrm{a}$ & $n / a$ & $\mathrm{n} / \mathrm{a}$ & 0.796 & $n / a$ & 0.914 \\
\hline
\end{tabular}




\section{elsevier_PATREC_7027}

MVSC-CEV

0.884

0.884

0.885

0.826

0.821

0.940

$n / a$ means result not available

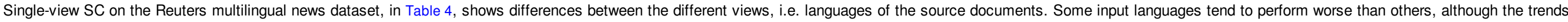

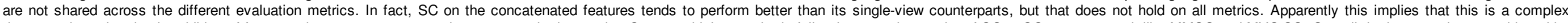

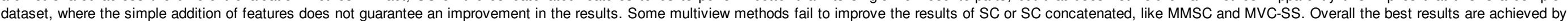
dataset, where the simple addition of features does not guarantee an improvement in
MVSC-CEV, except for the NMI value where MVC-JNMF produces the highest result.

Table 4 Clustering performance on the Reuters multilingual dataset.

$$
\text { alt-text: Table } 4
$$

\begin{tabular}{|c|c|c|c|c|c|c|}
\hline Method & F-score & Precision & Recall & NMI & Rand tindex & Purity \\
\hline SC view 1 & 0.365 & 0.315 & 0.435 & 0.310 & 0.154 & 0.531 \\
\hline SC view 2 & 0.288 & 0.219 & 0.421 & 0.303 & 0.110 & 0.549 \\
\hline SC view 3 & 0.376 & 0.340 & 0.420 & 0.304 & 0.181 & 0.569 \\
\hline SC view 4 & 0.296 & 0.216 & 0.471 & 0.312 & 0.201 & 0.538 \\
\hline SC view 5 & 0.336 & 0.270 & 0.445 & 0.308 & 0.193 & 0.568 \\
\hline SC concat. & 0.379 & 0.316 & 0.473 & 0.342 & 0.162 & 0.550 \\
\hline CotrainSC & 0.412 & 0.369 & 0.467 & 0.388 & 0.279 & $\mathrm{n} / \mathrm{a}$ \\
\hline MMSC & $\mathrm{n} / \mathrm{a}$ & $\mathrm{n} / \mathrm{a}$ & $\mathrm{n} / \mathrm{a}$ & 0.134 & $\mathrm{n} / \mathrm{a}$ & 0.390 \\
\hline MVC-SS & $\mathrm{n} / \mathrm{a}$ & $\mathrm{n} / \mathrm{a}$ & $\mathrm{n} / \mathrm{a}$ & $\mathrm{n} / \mathrm{a}$ & $\mathrm{n} / \mathrm{a}$ & 0.531 \\
\hline MVC-JNMF & $\mathrm{n} / \mathrm{a}$ & $\mathrm{n} / \mathrm{a}$ & $\mathrm{n} / \mathrm{a}$ & 0.409 & 0.535 & $\mathrm{n} / \mathrm{a}$ \\
\hline MVSC-BG & $\mathrm{n} / \mathrm{a}$ & $\mathrm{n} / \mathrm{a}$ & $\mathrm{n} / \mathrm{a}$ & 0.357 & $\mathrm{n} / \mathrm{a}$ & 0.577 \\
\hline MVSC-CEV & 0.459 & 0.394 & 0.550 & 0.341 & 0.536 & 0.619 \\
\hline
\end{tabular}

n/a means result not available.

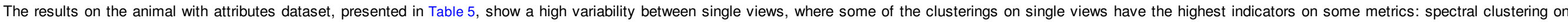

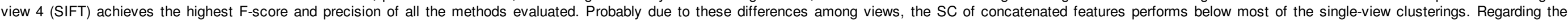
multiview methods, there is also a high variability, with MVSC-CEV obtaining the highest recall, NMI, Rand Index and purity values.

Table 5 Clustering performance on the animal with attributes (AWA) dataset.

alt-text: Table 5

\begin{tabular}{|c|c|c|c|c|c|c|}
\hline Method & F-score & Precision & Recall & NMI & Rand tindex & Purity \\
\hline SC view 1 & 0.162 & 0.124 & 0.233 & 0.359 & 0.544 & 0.273 \\
\hline SC view 2 & 0.485 & 0.455 & 0.519 & 0.742 & 0.774 & 0.593 \\
\hline SC view 3 & 0.124 & 0.108 & 0.145 & 0.291 & 0.552 & 0.235 \\
\hline SC view 4 & 0.550 & 0.521 & 0.583 & 0.796 & 0.778 & 0.647 \\
\hline SC view 5 & 0.353 & 0.282 & 0.473 & 0.644 & 0.659 & 0.439 \\
\hline SC view 6 & 0.451 & 0.374 & 0.567 & 0.743 & 0.768 & 0.566 \\
\hline SC concat. & 0.314 & 0.280 & 0.358 & 0.553 & 0.563 & 0.460 \\
\hline MMSC & $\mathrm{n} / \mathrm{a}$ & $\mathrm{n} / \mathrm{a}$ & $\mathrm{n} / \mathrm{a}$ & 0.698 & 0.585 & $\mathrm{n} / \mathrm{a}$ \\
\hline
\end{tabular}


n/a means result not available.

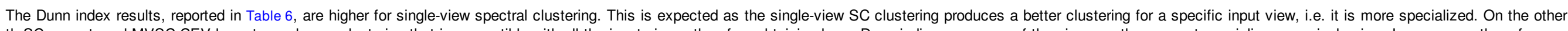

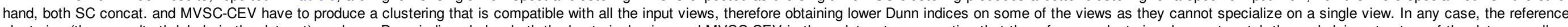

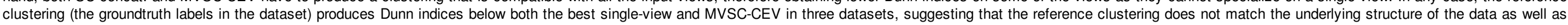
the clusterings produced by the different methods.

Table 6 Dunn index on the four data sets.

\section{alt-text: Table 6}

Method

\section{Reference}

\section{SC view 1}

\section{SC view 2}

\section{$\mathrm{SC}$ view 3}

SC view 4

SC view 5

SC view 6

SC concat

MVSC-CEV

\section{Digits}

\begin{tabular}{|l|l|}
\hline $0.823 \pm 0.267$ \\
\hline $0.770 \pm 0.200$ \\
\hline $0.797 \pm 0.213$ \\
\hline $0.932 \pm 0.169$ \\
\hline $0.977 \pm 0.179$ \\
\hline $0.747 \pm 0.190$ \\
\hline $0.804 \pm 0.146$ \\
\hline $0.902 \pm 0.160$ \\
\hline $0.846 \pm 0.202$ \\
\hline
\end{tabular}

\section{BBC}

$0.996 \pm 0.006$
$1.003 \pm 0.009$
$1.006 \pm 0.006$
-
-
-
-
$0.999 \pm 0.006$
$1.003 \pm 0.007$

\section{Reuters}

\begin{tabular}{|l|}
\hline $0.625 \pm 0.018$ \\
\hline $0.498 \pm 0.016$ \\
\hline $0.771 \pm 0.176$ \\
\hline $0.684 \pm 0.029$ \\
\hline $0.868 \pm 0.126$ \\
\hline $0.665 \pm 0.030$ \\
\hline- \\
\hline $0.743 \pm 0.050$ \\
\hline $0.826 \pm 0.049$
\end{tabular}

AWA

\begin{tabular}{|l|l|}
\hline $0.525 \pm 0.117$ \\
\hline $0.312 \pm 0.188$ \\
\hline $0.482 \pm 0.146$ \\
\hline $0.443 \pm 0.110$ \\
\hline $0.363 \pm 0.229$ \\
\hline $0.415 \pm 0.124$ \\
\hline $0.505 \pm 0.157$ \\
\hline $0.428 \pm 0.136$ \\
\hline $0.295 \pm 0.143$ \\
\hline
\end{tabular}

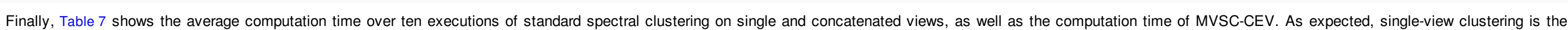

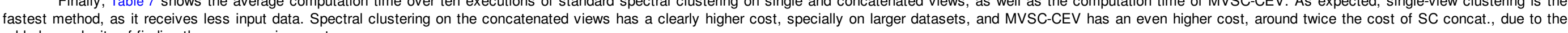
added complexity of finding the common eigenvectors.

Table 7 Execution times (seconds) on the four data sets.

alt-text: Table 7

\begin{tabular}{|c|c|c|c|c|}
\hline Method & Digits & $\mathrm{BBC}$ & Reuters & AWA \\
\hline SC single-view & $21.3 \pm 1.4$ & $10.5 \pm 0.0$ & $891.8 \pm 46.0$ & $1331.3 \pm 126.9$ \\
\hline SC concat. & $27.6 \pm 1.4$ & $16.5 \pm 0.8$ & $2017.2 \pm 97.4$ & $3886.7 \pm 415.2$ \\
\hline MVSC-CEV & $77.5 \pm 2.6$ & $18.2 \pm 0.7$ & $3590.3 \pm 107.5$ & $8167.5 \pm 649.3$ \\
\hline
\end{tabular}

\section{Conclusions}

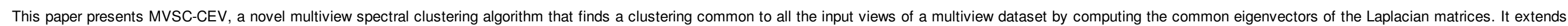

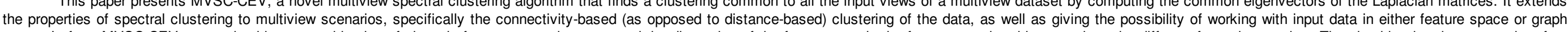

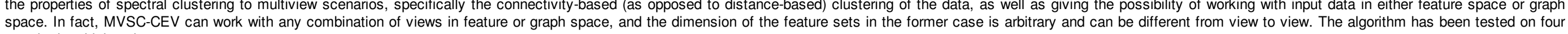
space. In fact, MVSC-CEV

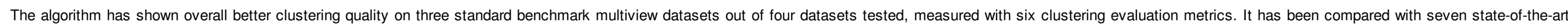

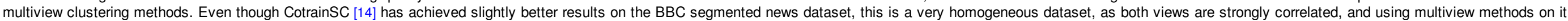




\section{elsevier_PATREC_7027}

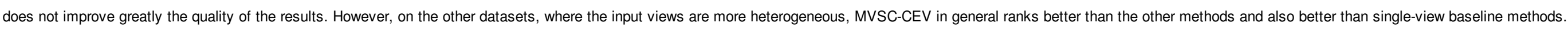

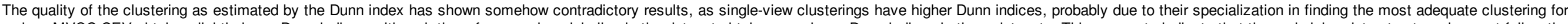

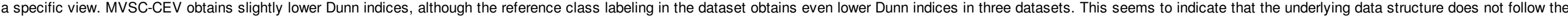
class assignment very closely.

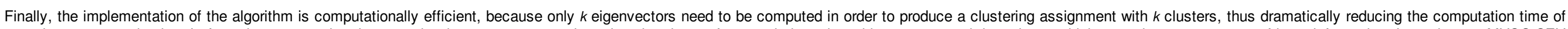

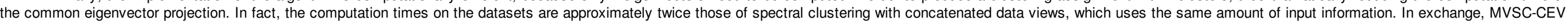
produces clearly better clusterings on almost all dataset and metric combinations.

\section{Acknowledgements}

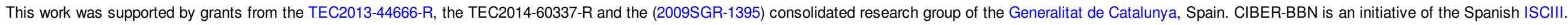

\section{References}

[1]

M. Amini, N. Usunier and C. Goutte, Learning from multiple partially observed views - an application to multilingual text categorization, Adv. Neural Inf. Process. Syst. 22, 2009, 28-36.

[2]

M. Breukelen, R.P.W. Duin, D.M.J. Tax and J.E. Hartog, Handwritten digit recognition by combined classifiers, Kybernetika 34 (4), 1998, [381]-386.

[3]

X. Cai, F. Nie and H. Huang, Multi-view K-means clustering on big data, IJCAI International Joint Conference on Artificial Intelligence, 2013, 2598-2604.

[4]

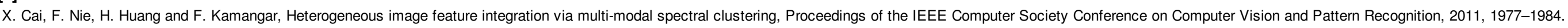

[5]

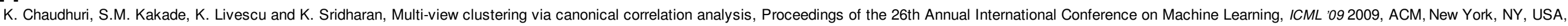
129-136.

[6]

Y. Cui, X.Z. Fern and J.G. Dy, Non-redundant multi-view clustering via orthogonalization, Seventh IEEE International Conference on Data Mining, ICDM 20072007, 133-142.

[7]

J.C. Dunn, Well-separated clusters and optimal fuzzy partitions, J. Cybern. 4 (1), 1974, 95-104

[8]

B.N. Flury, Common principal components in k groups, J. Am. Stat. Assoc. 79 (388), 1984, 892-898.

[9]

J. Gao, J. Han, J. Liu and C. Wang, Multi-view clustering via joint nonnegative matrix factorization., SDM, 2013, SIAM, 252-260.

[10]

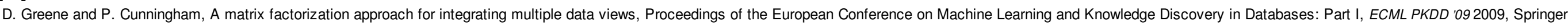
Verlag, Berlin, Heidelberg, 423-438.

[11]

T. Hofmann, Probabilistic latent semantic indexing, Proceedings of the 22nd Annual International ACM SIGIR Conference on Research and Development in Information Retrieval, 1999, ACM, 50-57.

[12]

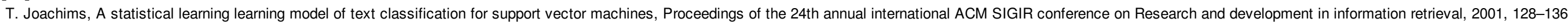
[13]

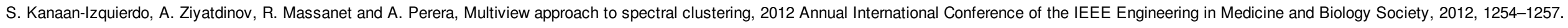

[14]

A. Kumar and H. Daumé III, A co-training approach for multi-view spectral clustering, International Conference on Machine Learning (ICML), 2011, 393-400.

[15]

A. Kumar, P. Rai and H. Daumé III, Co-regularized multi-view spectral clustering, Neural Information Processing Systems (NIPS), 2011, $1413-1421$. 


\section{elsevier_PATREC_7027}

[16]

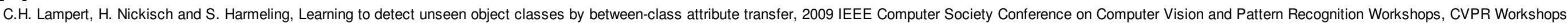
2009, 2009, 951-958.

[17]

Y. Li, F. Nie, H. Huang and J. Huang, Large-scale multi-view spectral clustering with bipartite graph, Twenty-Ninth AAAI Conference on Artificial Intelligence, 2015.

[18]

U. Luxburg, A tutorial on spectral clustering, Stat. Comput. 17 (4), 2007, 395-416.

[19]

C.D. Manning, P. Raghavan and H. Schütze, Introduction to information retrieval, J. Am. Soc. Inf. Sci. Technol. 1, 2008, 496.

[20]

A.Y. Ng, M.I. Jordan and Y. Weiss, On spectral clustering: analysis and an algorithm, Adv. Neural Inf. Process. Syst. 2001, 849-856.

[21]

V.R. de Sa, Spectral clustering with two views, ICML (International Conference on Machine Learning) Workshop on Learning with Multiple Views, $2005,20-27$.

[22]

J. Shi and J. Malik, Normalized cuts and image segmentation, Pattern Anal. Mach. Intell. IEEE Trans. 22 (8), 2000, 888-905.

[23]

G.W. Stewart and H.B. Jovanovich, Matrix perturbation theory, Math. Comput. Simul. 33 (1), 1991, 74.

[24]

N.T. Trendafilov, Stepwise estimation of common principal components, Comput. Stat. Data Anal. 54 (12), 2010, 3446-3457.

[25]

Vega-Pons and Ruiz-Shulcloper, A survey of clustering ensemble algorithms, Int. J. Pattern Recognit Artif Intell. 25 (03), 2011, 337-372.

[26]

H. Wang, F. Nie and H. Huang, Multi-view clustering and feature learning via structured sparsity, Proceedings of the 30th International Conference on Machine Learning (ICML-13), 28, 2013, 352-360.

[27]

X. Zhao, N. Evans and J.-L. Dugelay, A subspace co-training framework for multi-view clustering, Pattern Recognit. Lett. 41 (0), $2014,73-82$.

\section{Footnotes}

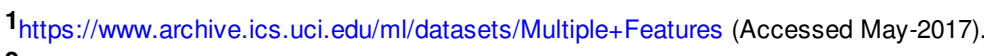

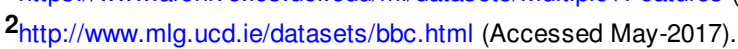

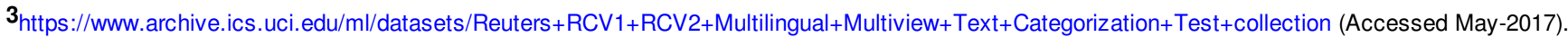

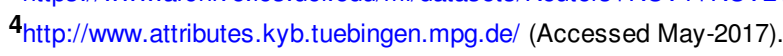

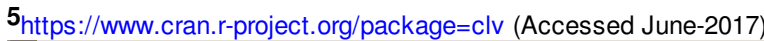

1

Highlights

- Multiview spectral clustering algorithm.

- Computes the common eigenvectors of the data similarity Laplacian matrices]

- Generic: any number, size and kind of views (both feature and graph space))

- Better overall performance than existing methods, tested on standard multiview data sets.

- Optimized method: to find $k$ clusters only needs to compute $k$ common eigenvectors_.

\section{Queries and Answers}




\section{elsevier_PATREC_7027}

Query: Please confirm that givennames and surnames have been identified correctly. Answer: Yes 